\title{
Why the Days Seem Shorter as We Get Older
}

\author{
A D R I A N B E J A N \\ Department of Mechanical Engineering and Materials Science, Duke University, \\ Durham, North Carolina 27708-0300, USA. Email: abejan@duke.edu
}

\begin{abstract}
Why does it feel that the time passes faster as we get older? What is the physical basis for the impression that some days are slower than others? Why do we tend to focus on the unusual (the surprise), not on the ever present? This article unveils the physics basis for these common observations. The reason is that the measurable 'clock time' is not the same as the time perceived by the human mind. The 'mind time' is a sequence of images, i.e. reflections of nature that are fed by stimuli from sensory organs. The rate at which changes in mental images are perceived decreases with age, because of several physical features that change with age: saccades frequency, body size, pathways degradation, etc. The misalignment between mental-image time and clock time serves to unite the voluminous observations of this phenomenon in the literature with the constructal law of evolution of flow architecture, as physics.
\end{abstract}

\section{Perceptions}

Among the most common human perceptions is that time passes faster as an individual becomes older. The days become shorter, and so do the years. We all have stories of this kind, from the long days of childhood and the never-ending class hours in elementary school, to days, months and years that now pass in a blur. The most common sayings convey this impression: Times flies; Where did the time go?; Last year was yesterday; Growing up took forever; A watched pot never boils; etc.

More subtle, and worth questioning is the impression that some days appear to pass more slowly than others. The 'slower' days are full of productivity, events, and memories of what happened. If you did not notice this difference between slow days and fast days, then you should pay attention to it, because in this difference lies the explanation for the lifelong puzzle sketched in the preceding paragraph. The hint is that productive days happen when the body and mind are rested, after periods of regular sleep, when in the morning you look in the mirror and you see a younger you, not a tired you.

Athletes learn the hard way the correlation between good rest and the speed of the passing time. Lack of rest makes you miss plays, unable to anticipate, unable to see the ball before it arrives. While sleep walking, the game is over before you know it. 
Young students learn the same physical truth while taking exams during a fixed time interval. The rested mind has more time to go through the problems, to find mistakes, to go back to the beginning, and try again. Lack of sleep, due to cramming the night before the exam, makes the time pass faster during the exam period. Cramming does not pay, but rest does, which is why the good coach rests the team before the big game.

Here is why this is important to you, the reader. Today, many young people experience time distortion because they spend too much time on social media. This has serious consequences, ranging from sleep deprivation to mood changes and mental disorder. This is why an understanding of the physics basis of how humans perceive the passing of time is essential.

\section{Physics}

Time represents perceived changes in stimuli (observed facts), such as visual images. ${ }^{1,2}$ The human mind perceives reality (nature, physics) through images that occur as visual inputs reach the cortex. The mind senses 'time change' when the perceived image changes. The time arrow in physics is the goal-oriented sequence of changes in flow configuration, the direction dictated by the constructal law. ${ }^{1-11}$ The present is different from the past because the mental viewing has changed, not because somebody's clock rings.

The 'clock time' that unites all the live flow systems, animate and inanimate, is measurable. The day-night period lasts 24 hours on all watches, wall clocks and bell towers. Yet, physical time is not mind time. The time that you perceive is not the same as the time perceived by another. Why? Because the young mind receives more images during one day than the same mind in old age. Said another way, if the lifespan is measured in terms of the number of images perceived during life, then the frequency of mental images at young age is greater than in old age (Figure 1). Here is why this should be:

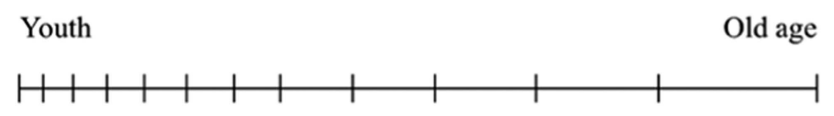

Perceived changes in images

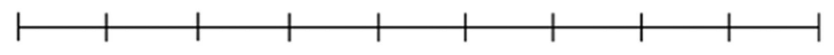

Clock time units

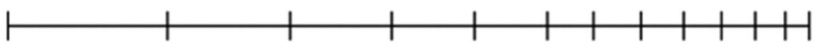

Perceived duration of one time unit

Figure 1. The misalignment between perceived time and clock time during lifetime. 
The sensory inputs that travel into the human body to become mental images 'reflections' of reality in the human mind - are intermittent. They occur at certain time intervals $\left(t_{1}\right)$, and must travel the body length scale $(L)$ with a certain speed $(V)$. In the case of vision, $t_{1}$ is the time interval between successive saccades. The time required by one mental image to travel from a sensory organ to the cortex is of order $t_{2} \sim L / V$. During life, the body length scale $(L)$ increases in proportion with the body mass $M$ raised to the power $1 / 3$, and, like all growth phenomena, the body mass increases over time in S-curve fashion, ${ }^{12}$ monotonically, slow - fast - slow, cf. Figure 2.
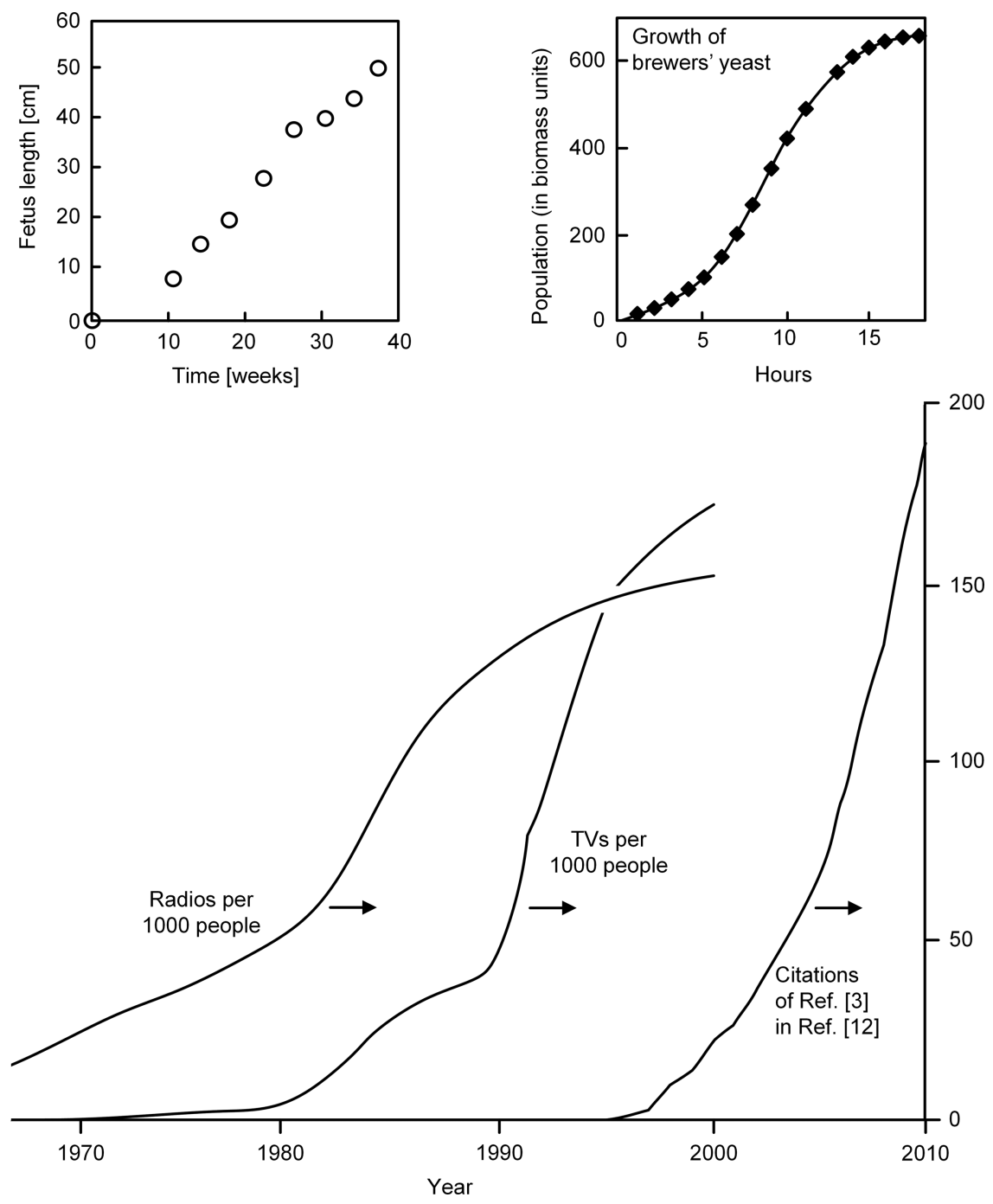

Figure 2. All growth phenomena (spreading, collecting) exhibit an S-shaped history curve $^{8}$ : four flow systems where the size of the flow space increases monotonically, slow-fast-slow. 


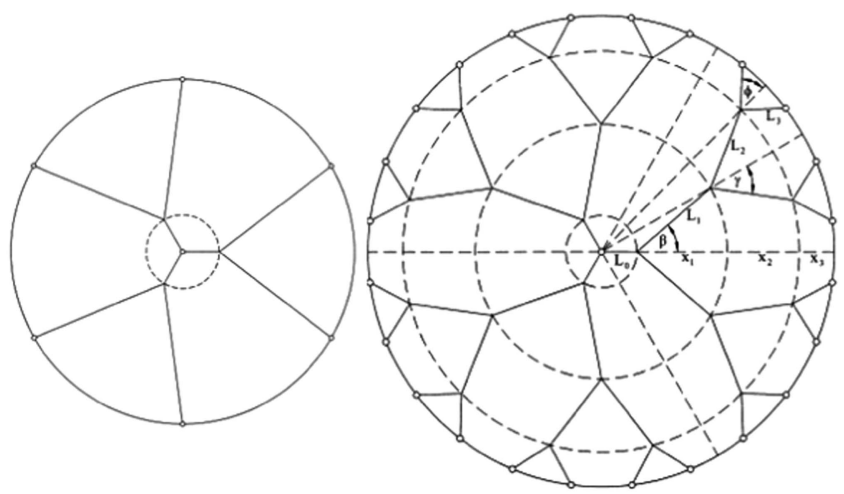

Figure 3. The length of the flow path increases as the body size and complexity increase. $^{9}$

The length traveled by inputs from external sensors to the cortex is actually greater than $L$, and it increases with age. The reason is that the complexity of the flow path needed by one signal to reach one point on the cortex increases as the brain grows and the complexity of the tree-shaped flow paths increase, cf. Figure $3 .^{13}$

The broad trend then is that $L$ increases with age. At the same time, $V$ decreases because of the ageing (degradation) of the flow paths. The key feature is that the physical time (the combined effect of $t_{1}$ and $t_{2}$ ) required by the occurrence of one mental image increases monotonically during the life of the individual. The frequency of mental images decreases monotonically, and non-uniformly (i.e. not at constant rate). This trend is illustrated qualitatively in Figure 1. Two summarizing conclusions follow.

(i) More of the recorded mental images should be from youth.

(ii) The 'speed' of the time perceived by the human mind should increase over life. The rate at which the physical time clock 'ticks' during one change in the mental image increases with age.

\section{Review}

The misalignment of the clock ticks and the changes perceived by the mind (Figure 1) brings together numerous observations and measurements accumulated in the literature, especially in the study of vision and cognition.

First, to define the terms, Fischer and Weber explain that during natural viewing conditions a normal adult subject makes 3-5 saccades in a second separated by periods of 200-300 ms during which the eyes do not make large or fast movements. ${ }^{14}$ These periods are usually called 'fixations'. If the retinal image, as a whole, is prevented from moving (by successful voluntary attempts not to move the eyes, or by technical means), vision rapidly becomes blurred and the perception of the retinal image fades away completely within 10 seconds. Fischer and Weber ${ }^{14}$ explain that the highly inhomogeneous structure of the primate retina, with an extremely high density of receptor and ganglion cells in the center, a specialized fovea, and a rapid decline of the cell densities toward the periphery, makes it almost impossible to have a 
homogeneous and simultaneous percept of the total visual field without somehow moving the fovea to different positions and acquiring and integrating information from these successive 'looks'. The existence of a fovea requires both eye movements and periods of fixation, that is, the active suppression of saccadic eye movements.

Although the reaction times of saccades is relatively stable $(200-250 \mathrm{~ms}),{ }^{15}$ the infant fixation times are shorter than in adults. In primates, there is a constant relationship between the duration, peak velocity and amplitude of saccadic eye movement, ${ }^{16-18}$ known as the 'main sequence', in which saccade trajectories have evolved toward optimizing the trade-off between accuracy and duration (speed) of the eye movement. This is also in accord with the physics basis for the human preference for displays shaped in 'golden-ratio' rectangular frames, which is the shape that is scanned the fastest by the two human eyes. ${ }^{2}$ As a result of an interaction between afferent, central and efferent neural processes we perceive a complete and stable visual field, which can serve as a frame within which we see motion and within which we move ourselves or parts of our body. ${ }^{14}$

Bahill and Stark showed that fatigue can produce overlapping saccades in which the high-frequency saccadic bursts should show large pauses, glissades in which the high-frequency bursts should be much shorter than appropriate for the size of the intended saccades, and low-velocity, long-duration, non-Main Sequence saccades in which the mononeuronal bursts should be of lower frequency and longer duration than normal. ${ }^{19}$ When the saccadic eye movement system fatigues, saccades become slower, and the neurological control signal stratagem changes. The term fatigue is used in a broad sense, as it was by McFarland: 'a group of phenomena associated with impairment, or loss, of efficiency and skill' ${ }^{20}$

The intuitive view that the world is processed as a seamless stream of ongoing perception has been challenged in the current literature. Herzog et al. discussed experimental evidence supporting the view that perception might be discrete, further supporting evidence for discrete theories. ${ }^{21}$ Visual information processing is similar to a sample and hold mechanism in engineering, as in analog/digital converters.

Herzog et al. also noted that the brain functions such that we consciously perceive only the most plausible solution, and not a confusing manifold of possibilities that occur during unconscious processing. ${ }^{21}$ The unconscious feature integration period is the period of sense-making. The discrete conscious perception is followed by unconscious processing over time. These two modes of absorbing inputs from the surroundings are analogous to all other flows from point (e.g. eye) to volume (e.g. brain). ${ }^{1}$ Observing fast and then letting it sink in slowly, is the same dynamic flow design as the long and fast, and short and slow that inhabits all nature, ${ }^{1}$ animate and inanimate. Conscious perception and the unconscious processing that follows are the 'invasion' and 'consolidation' phases of the universal S-curve phenomenon. ${ }^{12}$

Cicchini et al. review the classical model of time perception, which considers a single centralized clock that ticks at a constant rate. ${ }^{22}$ They point out that much experimental evidence seems to cast doubt on this model. The ability to pay 'attention' could modulate the tick rate and hence the duration of the events. ${ }^{23}$ Many studies found that the most surprising stimulus within a train of events is perceived 
longer, probably because it engages more transient attention or because the event is less predictable. Bruno et al. show that the apparent duration of moving visual objects is greater at higher than at lower speeds. ${ }^{24}$

Pöppel et al. argued that cognitive processes cannot be understood without their temporal dynamics; furthermore, certain logistical problems the brain has to deal with require an understanding of temporal processing. ${ }^{25}$ Eagleman et al. discussed the flashlag illusion, where a flash and a moving object in the same location appear to be offset. $^{26}$ They proposed an alternative in which visual awareness is neither predictive nor online but is postdictive, so that the percept attributed to the time of the flash is a function of events that happen in the $\sim 80 \mathrm{~ms}$ after the flash. Interpolation of the past is the only framework that provides a unified explanation for the flash-lag phenomenon.

VanRullen and Koch reconciled the unduly abandoned topic of discrete perception with current views and advances in neuroscience. ${ }^{27}$ Hainline et al. showed that for both infants and adults, linear relationships were found between the peak velocities of fast eye movements and their amplitudes (main sequences). ${ }^{28}$

With regard to the effect of aging, Sharpe and Zackon investigated horizontal saccades in young, middle-aged and elderly normal subjects. ${ }^{29}$ Saccades were elicited in response to three target conditions: predictable amplitude direct and timing; unpredictable amplitudes and directions at regular intervals; and unpredictably timed targets of predictable amplitude and direction. Peak velocities were significantly reduced in the elderly when target amplitude and direction were predictable. Latencies were prolonged in the elderly under all conditions. Saccadic accuracy was significantly decreased in elderly subjects. Support for the thesis that age-related cognitive slowing is global is provided by Myerson et al. ${ }^{30}$ Although older adults perform worse than younger adults in complex decision-making scenarios, prior experience should be taken into account in aging studies. ${ }^{31}$

\section{Conclusion}

Summing up, we conclude that the perceived misalignment between mental-image time and clock time (Figure 1) is in accord with and unifies the growing number of observations that describe aspects of this phenomenon in the literature. The physics basis is captured by the constructal law of evolution in nature (see Figures 2 and 3).

\section{Acknowledgement}

Professor Adrian Bejan's research was supported by the US National Science Foundation.

\section{References}

1. A. Bejan (2016) The Physics of Life (New York: St. Martin's Press).

2. A. Bejan (2009) The golden ratio predicted: Vision, cognition and locomotion as a single design in nature. International Journal of Design \& Nature and Ecodynamics, 4(2), pp. 97-104. 
3. T. Basak (2013) The law of life: The bridge between Physics and Biology. Physics of Life Reviews, 8, pp. 249-252.

4. A.F. Miguel (2011) The physics principle of the generation of flow configuration. Physics of Life Reviews, 8(3), pp. 243-244.

5. A.H. Reis (2011) Design in nature, and the laws of physics. Physics of Life Reviews, 8(3), pp. 255-256.

6. L. Wang (2011) Universality of design and its evolution. Physics of Life Reviews, 8(3), pp. 257-258.

7. G. Lorenzini and C. Biserni (2011) The constructal law: From design in nature to social dynamics and wealth as physics. Physics of Life Reviews, 8(3), pp. 259-260.

8. U. Lucia, A. Ponzetto and T.S. Deisboeck (2014) A thermo-physical analysis of the proton pump vacuolar-ATPase: The constructal approach. Scientific Reports, 4, p. 6763.

9. U. Lucia, G. Grazzini, B. Montrucchio, G. Grisolia, R. Borchiellini, G. Gervino, C. Castagnoli, A. Ponzetto and F. Silvagno (2015) Constructal thermodynamics combined with infrared experiments to evaluate temperature differences in cells. Scientific Reports, 5, p. 11587.

10. U. Lucia, A. Ponzetto and T.S. Deisboeck (2016) Constructal approach to cell membranes transport: Amending the 'Norton-Simon' hypothesis for cancer treatment. Scientific Reports, 6, p. 19451.

11. A. Bejan (2012) Why the bigger live longer and travel farther: Animals, vehicles, rivers and the winds. Scientific Reports, 2, no. 594.

12. A. Bejan and S. Lorente (2011) The constructal law origin of the logistics $\mathrm{S}$ curve. Journal of Applied Physics, 110, 024901.

13. W. Wechsatol, S. Lorente and A. Bejan (2002) Optimal tree-shaped networks for fluid flow in a disc-shaped body. International Journal of Heat and Mass Transfer, 45, pp. 4911-4924.

14. B. Fischer and H. Weber (1993) Express saccades and visual attention. Behavioral and Brain Sciences, 16, pp. 553-610.

15. K.M. Butler, R.T. Zacks and J.M. Henderson (1999) Suppression of reflexive saccades in younger and older adults. Memory \& Cognition, 27(4), pp. 584-591.

16. D. Boghen, B.T. Troost, R.B. Daroft, L.F. Dell'Osso and J.E. Birkett (1974) Velocity characteristics of normal human saccades. Investigative Ophthalmology, 13(8), pp. 619-623.

17. C.M. Harris, L. Hainline, L. Abramov, E. Lemerise and C. Camenzuli (1998) The distribution of fixation durations in infants and naïve adults. Vision Research, 28(3), pp. 419-432.

18. C.M. Harris and D.M. Wolpert (2006) The main sequence of saccades optimizes speed-accuracy trade-off. Biological Cybernetics, 95, pp. 21-29.

19. A.T. Bahill and L. Stark (1975) Overlapping saccades and glissades are produced by fatigue in the saccadic eye movement system. Experimental Neurology, 48, pp. $95-106$.

20. R.A. McFarland (1971) Understanding fatigue in modern life. Ergonomics, 14, pp. 1-10.

21. M.H. Herzog, T. Kammer and F. Scharnowski (2016) Time slices: What is the duration of a percept? PLOS Biology, 12 April 2016. DOI: 10.1371/journal. pbio. 1002433 .

22. G.M. Cichini and M.C. Morrone (2009) Shifts in spatial attention affect the perceived duration of events. Journal of Vision, 9(1), pp. 1-13. 
23. I. Levin and D. Zakay (Eds) (1989) Subjective time and attentional resource allocation: An integrated model of time estimation. Time and Human Cognition. A Life-span Perspective (Amsterdam: North-Holland), pp. 365-397.

24. A. Bruno, I. Ayhan and A. Johnston (2015) Changes in apparent duration following shifts in perpetual timing. Journal of Vision, 15(6), pp. 1-18.

25. E. Pöppel (1997) A hierarchical model of temporal perception. Trends in Cognitive Sciences, 1(2), p. 56.

26. D.M. Eagleman and T.J. Sejnowski (2000) Motion integration and postdiction in visual awareness. Science, 287, pp. 2036-2038.

27. R. VanRullen and C. Koch (2003) Is perception discrete or continuous? TRENDS in Cognitive Sciences, 7(5), pp. 207-213.

28. L. Hainline, J. Turkel, I. Abramov, E. Lemerise and C.M. Harris (1984) Characteristics of saccades in human infants. Vision Research, 24(12), pp. 1771-1780.

29. J.A. Sharpe and D.H. Zackon (1987) Senescent saccades. Acta Otolaryngol (Stockh), 104, pp. 422-428.

30. J. Myerson, S. Hale, D. Wagstaff, L.W. Poon and G.A. Smith (1990) The information-loss model: A mathematical theory of age-related cognitive slowing. Psychological Review, 97(4), pp. 475-487.

31. N.J. Blanco, B.C. Love, M. Ramscar and A.R. Otto (2016) Exploratory decision-making as a function of lifelong experience, not cognitive decline. Journal of Experimental Psychology: General, 145(3), pp. 284-297.

\section{About the Author}

Adrian Bejan is the J.A. Jones Distinguished Professor at Duke University, and is a member of Academia Europaea. He is a graduate of the Massachusetts Institute of Technology (BS 1971, MS 1972, PhD 1975) and a recipient of the Benjamin Franklin Medal (2018). He has received 18 honorary doctorates from universities in 11 countries. He has published widely on thermodynamics, evolution, and the constructal law of organization in nature, as physics. 